\title{
Bifidobacteria and lactobacilli in the gut microbiome of children with non-alcoholic fatty liver disease: which strains act as health players?
}

Valerio Nobilii, ${ }^{1,2}$, Lorenza Putignani ${ }^{3,4}$, Antonella Mosca ${ }^{1}$, Federica Del Chierico ${ }^{4}$, Pamela Vernocchi $^{4}$, Anna Alisi², Laura Stronati ${ }^{5}$, Salvatore Cucchiara ${ }^{6}$, Marco Toscano $^{7}$, Lorenzo Drago ${ }^{7}$

\author{
${ }^{1}$ Hepato-Metabolic Disease Unit, “Bambino Gesū” Children's Hospital, IRCCS, Rome, \\ Italy \\ ${ }^{2}$ Liver Research Unit, “Bambino Gesū” Children's Hospital, IRCCS, Rome, Italy \\ 3Unit of Parasitology, Bambino Gesù Children's Hospital, IRCCS, Rome, Italy \\ ${ }^{4}$ Unit of Human Microbiome, Bambino Gesù Children's Hospital, IRCCS, Rome, Italy \\ ${ }^{5}$ Department of Cellular Biotechnology and Hematology, Sapienza University of Rome, \\ Italy \\ ${ }^{6}$ Pediatric Gastroenterology and Liver Unit, Department of Pediatrics, Sapienza \\ University, Rome, Italy \\ ${ }^{7}$ Laboratory of Clinical Chemistry and Microbiology, IRCCS Galeazzi Orthopaedic \\ Institute, Milan, Italy
}

Submitted: 9 May 2016

Accepted: 22 July 2016

Arch Med Sci 2018; 14, 1: 81-87

DOI: $10.5114 /$ aoms.2016.62150

Copyright $\odot 2016$ Termedia \& Banach

\section{Abstract}

Introduction: Non-alcoholic fatty liver disease (NAFLD), considered the leading cause of chronic liver disease in children, can often progress from non-alcoholic fatty liver (NAFL) to non-alcoholic steatohepatitis (NASH). It is clear that obesity is one of the main risk factors involved in NAFLD pathogenesis, even if specific mechanisms have yet to be elucidated. We investigated the distribution of intestinal bifidobacteria and lactobacilli in the stools of four groups of children: obese, obese with NAFL, obese with NASH, and healthy, age-matched controls (CTRLs).

Material and methods: Sixty-one obese, NAFL and NASH children and 54 CTRLs were enrolled in the study. Anthropometric and metabolic parameters were measured for all subjects. All children with suspected NASH underwent liver biopsy. Bifidobacteria and lactobacilli were analysed in children's faecal samples, during a broader, $16 \mathrm{~S}$ rRNA-based pyrosequencing analysis of the gut microbiome.

Results: Three Bifidobacterium spp. (Bifidobacterium longum, Bifidobacterium bifidum, and Bifidobacterium adolescentis) and five Lactobacillus spp. (L. zeae, L. vaginalis, L. brevis, L. ruminis, and L. mucosae) frequently recurred in metagenomic analyses. Lactobacillus spp. increased in NAFL, NASH, or obese children compared to CTRLs. Particularly, L. mucosae was significantly higher in obese $(p=0.02426)$, NAFLD $(p=0.01313)$ and NASH $(p=0.01079)$ than in CTRLs. In contrast, Bifidobacterium spp. were more abundant in CTRLs, suggesting a protective and beneficial role of these microorganisms against the aforementioned diseases.

Conclusions: Bifidobacteria seem to have a protective role against the development of NAFLD and obesity, highlighting their possible use in developing novel, targeted and effective probiotics.

Key words: paediatric non-alcoholic fatty liver disease, gut microbiota, Bifidobacterium, Lactobacillus.

\author{
Corresponding author: \\ Prof. Valerio Nobili MD, PhD \\ Hepato-Metabolic \\ Disease Unit \\ "Bambino Gesù" \\ Children's Hospital \\ IRCCS \\ Via S. Onofrio 4 \\ 00165 Rome, Italy \\ Phone: +390668592192 \\ E-mail: valerio.nobili@opbg.net
}




\section{Introduction}

Non-alcoholic fatty liver disease (NAFLD) is the most common cause of chronic liver disease in adults and children. It is regarded as a spectrum of hepatic conditions, which ranges from steatosis (non-alcoholic fatty liver - NAFL) to non-alcoholic steatohepatitis (NASH), with or without fibrosis [1]. The pathogenesis of NAFLD has been partially elucidated. It is generally accepted that NAFLD is pathogenically a "multiple-hit" disease. This hypothesis focuses on the central role of elevated circulating levels of free fatty acids (FFA) and insulin resistance that mutually interact to cause FFA accumulation in hepatocytes, oxidative stress and an inflammatory response, providing the grounds for progressive liver damage [2]. Interestingly, several studies have shown that gut microbiota may also play a key role in NAFLD development and progression [1]. Microbiota refers to the ecological community of commensals, symbiotic and pathogenic microorganisms that share our body space and internal cavities. The human microbiota consists of about 100 trillion microbial cells, outnumbering human cells 100 to 1 [3], with five phyla representing the majority of the community. There are approximately 160 species in the large intestine of any individual, and very few of these are shared between unrelated individuals. It has been demonstrated that microbiota composition can influence the proportion of calories absorbed from intestinal contents [4].

Indeed, bacteria play an important role in the absorption and emulsion of fat and fat-soluble vitamins in the small bowel. The gut microbiota may also produce endotoxins, such as lipopolysaccharide (LPS), that during impaired intestinal permeability may reach the circulation, leading to extraand intra-hepatic inflammatory responses, which, in turn, induce the progression from NAFL to NASH [1]. It is now recognised that the gut microbiota and chronic liver disease are closely linked. In addition, Ridaura et al. demonstrated a potential relationship between the gut microbiome and the development of an obese phenotype [5].

Recently, our group demonstrated that omics-based integrated charts of gut microbiota may profoundly assist in the understanding of correlations between enterogradients and NAFLD onset and progression, thus suggesting either phylome or metabolome indicators that may be used as phenome biomarkers of gut microbiota disease [6].

Furthermore, the gut microbiota is influenced by diet, age, infection, hygiene habits and antibiotic therapy [7]. Patients affected by NAFLD showed a high prevalence of small intestine bacterial overgrowth (SIBO) and increased intestinal permeability that improved bacterial translocation, exposing the liver to toxic microbial metabolites
$[8,9]$. Obese patients with NAFLD displayed a reduction of Bacteroidetes numbers and an increase in Firmicutes numbers compared to patients with normal weight [10]. Intriguingly, the loss of weight in response to the limited intake of carbohydrates and fats increased the prevalence of Bacteroidetes in the intestinal microbiota [10]. Furthermore, studies on mice with NAFLD have demonstrated that lactobacilli and other probiotic bacteria reduced plasma cholesterol concentrations and protected against fat accumulation in adipose tissue and hepatocytes [8, 11].

Understanding the nature of gut dysbiosis, the integrity of the intestinal barrier and mechanisms of the hepatic immune response to gut-derived factors is potentially relevant to the development of new therapies to treat chronic liver diseases [12]. The term "dysbiosis" was coined by Metchnikoff to describe altered pathogenic bacteria in the gut [13]. Dysbiosis was defined as qualitative and quantitative changes in the intestinal flora, their metabolic activity and their local distribution [14]. Recently, we demonstrated [15] that probiotic treatment in obese children with NAFLD reduced their body mass index (BMI) and hepatic steatosis as assessed by ultrasound, and increased circulating levels of glucagon-like peptide-1 (GLP-1), a potent hormone released from intestinal $L$ cells [16]. These encouraging results, also confirmed by other studies [17-19], suggest that improving our knowledge of bacterial species that live in the gut of NAFLD subjects may contribute significantly to allowing us to select the best probiotic(s) to strongly ameliorate liver damage.

In a recent, extensive characterisation of the gut microbiota profile of children with NAFLD compared to obese and lean children without liver disease, it emerged that Oscillospira is a marker of the healthy status of the liver, while Ruminococcus and Dorea increased concomitantly with NAFL towards NASH progression [6]. However, since the most commercially available probiotics are Bifidobacterium and Lactobacillus, the aim of the present study was to discuss these species in terms of the severity of disease in order to determine which can be used as treatment for children with NASH.

\section{Material and methods}

This study was conducted on 61 consecutive overweight or obese child patients (35 males, aged $11.45 \pm 2.42$ years) who were followed at the Hepato-Metabolic Disease Unit of Bambino Gesù Children's Hospital and Research Institute (OPBG; Rome, Italy) in 2013. All patients were tested for secondary causes of fatty liver such as hepatitis, cytomegalovirus and Epstein-Barr viral infections, liver disease, autoimmune disease, metabolic liver disease, Wilson's disease, coeliac disease or a de- 
ficiency of $\alpha_{1}$-antitrypsin, which were excluded using standard clinical, laboratory and histological criteria. Fifty-three patients, with $\mathrm{BMI}>95^{\text {th }}$ percentile, and/or hypertransaminasaemia and steatosis, underwent a liver biopsy for the evaluation of liver damage. The study population consisted of three groups: 8 (13.12\%) obese, 27 (44.26\%) with NAFLD and 26 (42.62\%) with definite NASH. Moreover, 54 healthy, age-matched children enrolled at the OPBG Human Microbiome Unit were used as controls (CTRLS).

The Hospital Ethics Research Committee approved the study, which was conducted in accordance with the Declaration of Helsinki (as revised in Seoul, Korea, October 2008). Written informed consent was obtained from the parents of the enrolled subjects. This study was approved by the OPBG Ethics Committee (protocol no. 768.12).

Faecal samples were collected from all affected children and CTRLs, and analysed at the OPBG Human Microbiome Unit.

\section{Anthropometric and laboratory evaluations}

Anthropometric parameters, including weight, height, BMI, and waist circumference, were measured in all children using standardised methods. Obesity was defined by a BMI $\geq 95^{\text {th }}$ percentile, adjusted for age and sex according to World Health Organization growth charts. Fasting plasma glucose (FPG), alanine aminotransferase (ALT), aspartate aminotransferase (AST), $\gamma$-glutamyltransferase (GGT), fasting serum triglycerides (TG), and total cholesterol were determined using standardised methods in our laboratory. Insulin was measured by means of a radioimmunoassay (MYRIA; Technogenetics, Milan, Italy). The degree of insulin sensitivity/resistance was determined by a homeostatic model assessment (HOMA). A HOMA value $>2$ was considered an indication of insulin resistance [20]. All patients underwent an abdominal ultrasound evaluation. Hepatic ultrasonography scanning by assigned and experienced radiologists, who were blinded to the health conditions of the patients, was performed on children after overnight fasting. The liver was graded for markers of hepatic steatosis by using established criteria: a bright hepatic echo pattern (compared with the echo response of the right kidney). All patient data have been previously reported by Del Chierico et al. (Table I from [6]).

\section{DNA extraction}

Every stool sample collected from the 115 children was processed for further 16S rRNA-targeted metagenomic analysis. From each sample, DNA was extracted using the QIAamp DNA Stool Mini Kit (Qiagen, Germany). To start, stool sam- ples were resuspended in $1.5 \mathrm{ml}$ of phosphatebuffered saline, homogenised by vortexing for $2 \mathrm{~min}$ and centrifuged at 20,800 $\times \mathrm{g}$. After the removal of supernatant, the pellet was resuspended in $500 \mu$ l of phosphate-buffered saline, together with $500 \mu \mathrm{l}$ of beads/PBS $(1 \mathrm{mg} / \mu \mathrm{l}$, [w/v]; glass beads, acid-washed Sigma-Aldrich, St. Louis, MI, USA). The supernatant was collected and treated for one freeze-thaw cycle $\left(-20^{\circ} \mathrm{C} / 70^{\circ} \mathrm{C}\right)$ for $20 \mathrm{~min}$ each step, as previously described. After centrifugation at $5200 \times \mathrm{g}$ for $5 \mathrm{~min}$, the supernatant was subjected to QIAamp DNA Stool Mini Kit (Qiagen, Hilden, Germany) extraction according to the manufacturer's instructions and then processed for metagenomics pipelines, as previously described [15]. The gut microbiome was investigated by pyrosequencing $V 1-V 3$ regions of the $16 \mathrm{~S}$ rRNA gene (amplicon size $520 \mathrm{bp}$ ) on a GS Junior platform (454 Life Sciences, Roche Diagnostics, Monza, Italy), according to the original pipelines described by Del Chierico et al. 2016 [6].

\section{Statistical analysis}

Univariate testing for differential abundances of each taxonomic unit between two or more groups was tested using a non-parametric Kruskal-Wallis $\mathrm{H}$ test.

\section{Open access data repository}

Bioprojects healthy children gut microbiota: PRJNA268064, PRJNA 268067, PRJNA268144, PRJNA268157, PRJNA268286 and PRJNA280490, Metaomic OPBG Consortium (http://www.ncbi. $\mathrm{n} / \mathrm{m}$.nih.gov/bioproject/?term=).

\section{Results}

Our observation, based on the study results reported previously [6], highlighted the prevalent distribution of three main Bifidobacterium spp. (B. longum, B. bifidum, and B. adolescentis) and five Lactobacillus spp. (L. zeae, L. vaginalis, L. brevis, $L$. ruminis, and $L$. mucosae) that variously recurred in targeted metagenomic analyses. In particular, L. zeae was present in all four groups (i.e., obese, NAFL and NASH children), while $L$. ruminis and $L$. vaginalis were present only in the NAFL and $\mathrm{NASH}$ groups. Lactobacillus mucosae was associated with obese and NAFL groups, and L. brevis with NASH and CTRL groups (Figure 1). Interestingly, Kruskal-Wallis $\mathrm{H}$ test performed among the groups revealed that $L$. mucosae was significantly higher in obese $(p=0.02426)$, NAFLD ( $p=$ $0.01313)$ and NASH $(p=0.01079)$ than in CTRLs.

In contrast, an inverse correlation between bifidobacteria and NAFL and NASH was observed; in particular, while $B$. longum, $B$. adolescentis and $B$. bifidum were shown to be strongly reduced in 
A

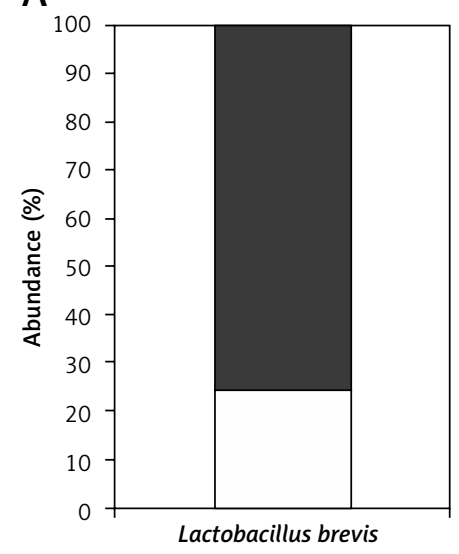

D

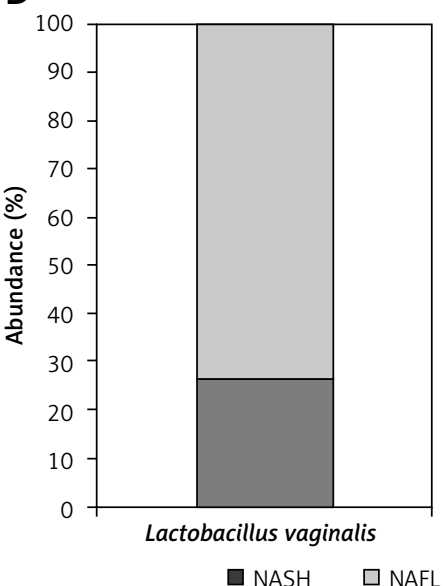

B

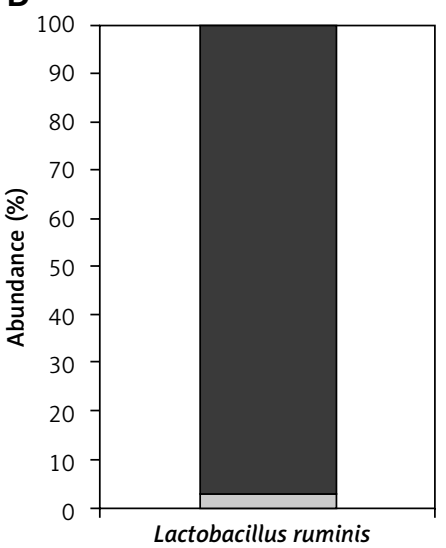

E

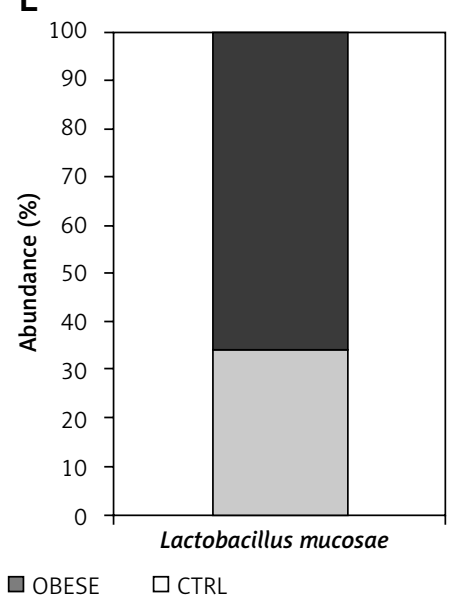

Figure 1. Histograms showing the percentage of relative abundances of Lactobacillus spp. distributed among the various groups of children. Abundances were calculated for: $\mathbf{A}-$ L. brevis; $\mathbf{B}-$ L. ruminis; $\mathbf{C}-L$. zeae; $\mathbf{D}-L$. vaginalis and $\mathrm{E}-$ L. mucosae

A

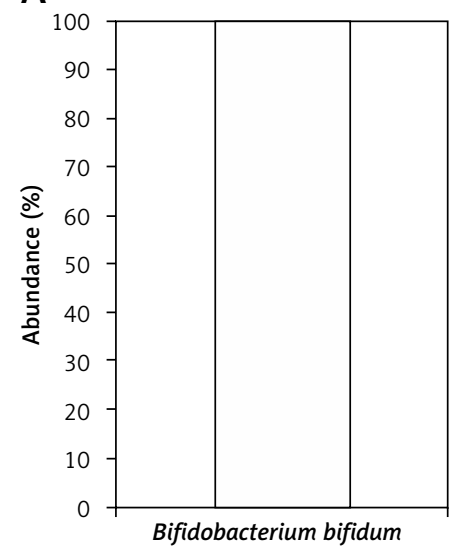

B

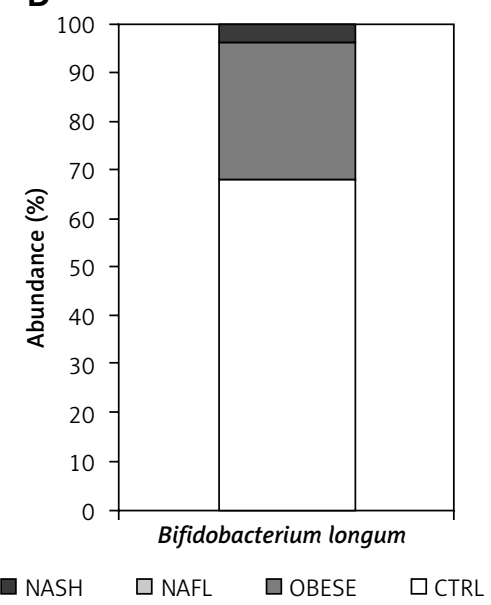

C

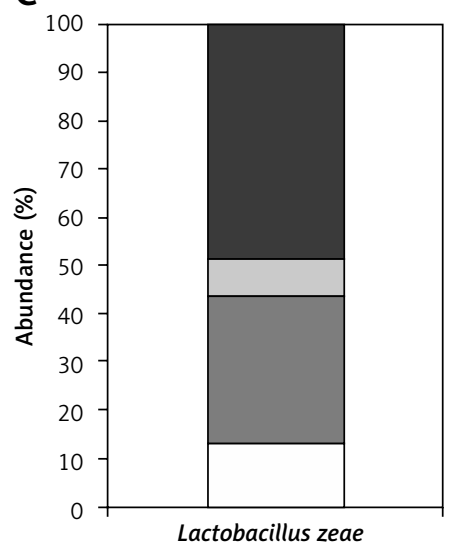


between a specific probiotic strain with each disorder [21-31]. Recently, new studies in systems biology have substantially supported the selection of specific, disease-targeted probiotics [6, 32].

In previous studies, bifidobacteria were able to decrease the NAFLD activity score by reducing hepatic exposure to intestinal products such as LPS that promote hepatic inflammatory cytokine production and oxidative stress, leading to liver injury [33, 34]. Bifidobacterium longum as a probiotic has been observed to reduce endotoxin levels, thus promoting reduced hepatocellular injury, portal and parenchymal inflammation, and NASH activity [34]. Ren et al. highlighted the ability of bifidobacteria to increase the hepatic expression of sirtuin $1 \alpha$ (SIRT1- $\alpha)$, which, in turn, up-regulated the expression of the peroxisome proliferator-activated receptor $\alpha$ (PPAR- $\alpha$ ) and down-regulated the level of the sterol regulatory element-binding protein 1c (SREBP-1c), thus preventing the progression of NAFLD [35].

In the present study, we also observed an increase of Lactobacillus spp. in NAFL and NASH patients compared to CTRLs. These data contrast with a previous experimental study demonstrating that the administration of a $L$. johnsonii-based probiotic to mice fed a high-fat diet (HFD) was able to prevent the development of NAFLD [35]. More specifically, the probiotic supplementation promoted an increase of lactobacilli and a decrease of bacteria from the Enterobacteriaceae family in the gut, providing preliminary protection. Moreover, it reduced both gut permeability and LPS serum levels, which are generally altered in mice with HFD-induced NAFLD. It is worth noting that dysfunctional gut permeability may allow liver exposure to gut-derived bacterial metabolites or antigens [36]. Similarly, gut microbiota-derived LPS leads to liver inflammation and oxidative stress [37]. Interestingly, Sohn et al. demonstrated that administering $L$. paracasei may prevent an increase in intestinal permeability in an NASH model by inhibiting the pro-inflammatory M1 Kupffer cell response and promoting the anti-inflammatory $M 2$ response [38]. The discrepancy between these results and our study may be attributable to strain-specific activity of lactobacilli or to the use of animal models that may not be fully representative of human physiology.

It is noteworthy that all obese patients enrolled in the present study showed a significant decrease in bifidobacteria abundance compared to CTRLs, thus also suggesting a possible role for bifidobacteria in this disease. Indeed, their role in obesity is controversial, some studies demonstrating an involvement in body weight gain, with others showing opposite results $[39,40]$. In particular, $B$. longum has been observed to significantly reduce body weight gain and fat weight in a group of mice fed for 5 weeks with a HFD [39]. Interest-

\begin{tabular}{|l|c|}
\hline \multicolumn{2}{|l|}{ Bifidobacteria and lactobacilli: good and bad for NAFLD } \\
\hline & GOOD/BAD \\
\hline Lactobacillus brevis & \\
\hline Lactobacillus ruminis & \\
\hline Lactobacillus zeae & \\
\hline Lactobacillus mucosae & \\
\hline Lactobacillus vaginalis & \\
\hline Bifidobacterium bifidum & \\
\hline Bifidobacterium longum & \\
\hline Bifidobacterium adolescentis & \\
\hline
\end{tabular}

Figure 3. Schematic representation of the beneficial and unfavourable effects of bifidobacteria and lactobacilli in paediatric NAFLD

ingly, the anti-obesity activity of probiotic microorganisms is often associated with a hypocholesterolaemic effect, through inhibition of cholesterol synthesis, elimination of cholesterol in faeces, absorption of cholesterol back into the body and inhibition of bile salt recycling [41, 42].

Lactobacillus spp. are associated both with normal weight and obesity; however, it is conceivable that these species, often found in the gut of obese subjects, may share with $L$. reuteri the same mechanism underlying weight gain: improvement of the intestine's ability to absorb and process nutrients, which increases food conversion and finally leads to body weight gain [43]. The hypocholesterolaemic properties of lactobacilli have already been reported. In fact, these bacteria increased high-density lipoprotein in serum and short-chain fatty acid (SCFA) concentrations in faeces, and promoted faecal bile acid excretion [44]. Moreover, some Lactobacillus spp. may exhibit antidiabetic activity, with a significant reduction of blood glucose levels seen in infected mice $[37,45]$.

In conclusion, we highlight that bifidobacteria and lactobacilli strains may differently impact NAFLD (Figure 3). Noticeably, we highlight that some Bifidobacterium spp. may have a protective role in the development of NASH, NAFL and obesity due to their marked reduction in patients with these disorders. Further studies should focus on assessing whether the administration of specific and well-selected B. adolescentis, B. longum and $B$. bifidum strains, alone or in combination with Oscillospira [6], may improve symptoms and the clinical course of NAFLD in obese children.

\section{Acknowledgments}

The authors wish to thank Alessandra Russo for her technical support and BioMed Proofreading LLC for proofreading service.

Financial Support: Integration of microbiota enterotypic profiles and phenomics data as new translational approaches for health control during 
infancy and for the treatment of complex phenotype diseases: the role of gut microbiota (Grant ID RC 201402G003251, Italian Ministry of Health) to LP; Model-Driven Paediatric European Digital Repository, European Commission under the FP7-ICT Programme to BDP; and Fondazione Luca Barbareschi.

\section{Conflict of interest}

The authors declare no conflict of interest.

\section{References}

1. Chalasani N, Younossi Z, Lavine JE, et al. The diagnosis and management of non-alcoholic fatty liver disease: practice guideline by the American Gastroenterological Association, American Association for the Study of Liver Diseases, and American College of Gastroenterology. Gastroenterology 2012; 142: 1592-609.

2. Polyzos SA, Kountouras J, Zavos C, Deretzi G. Nonalcoholic fatty liver disease: multimodal treatment options for a pathogenetically multiple-hit disease. J Clin Gastroenterol 2012; 46: 272-84.

3. Savage DC. Microbial ecology of the gastrointestinal tract. Annu Rev Microbiol 1977; 31: 107-33.

4. Turnbaugh PJ, Ley RE, Mahowald MA, Magrini V, Mardis ER, Gordon JI. An obesity-associated gut microbiome with increased capacity for energy harvest. Nature 2006; 444: 1027-31.

5. Ridaura VK, Faith JJ, Rey FE, et al. Gut microbiota from twins discordant for obesity modulate metabolism in mice. Science 2013; 341: 1241214.

6. Del Chierico F, Nobili V, Vernocchi P, et al. Gut microbiota profiling of pediatric NAFLD and obese patients unveiled by an integrated meta-omics based approach. Hepatology 2016; doi: 10.1002/hep.28572 [Epub ahead of print].

7. Putignani L, Del Chierico F, Petrucca A, Vernocchi P, Dallapiccola $B$. The human gut microbiota: a dynamic interplay with the host from birth to senescence settled during childhood. Pediatr Res 2014; 76: 2-10.

8. Ley RE, Turnbaugh PJ, Klein S, Gordon JI. Microbial ecology: human gut microbes associated with obesity. Nature 2006; 444: 1022-3.

9. Miele L, Valenza V, La Torre G, et al. Increased intestinal permeability and tight junction alterations in nonalcoholic fatty liver disease. Hepatology 2009; 49: 1877-87.

10. Zhu L, Baker SS, Gill C, et al. Characterization of gut microbiomes in nonalcoholic steatohepatitis (NASH) patients: a connection between endogenous alcohol and NASH. Hepatology 2013; 57: 601-9.

11. Kim SW, Park KY, Kim B, Kim E, Hyun CK. Lactobacillus rhamnosus GG improves insulin sensitivity and reduces adiposity in high-fat diet-fed mice through enhancement of adiponectin production. Biochem Biophys Res Commun 2013; 431: 258-63.

12. Boursier J, Mueller O, Barret M, et al. The severity of nonalcoholic fatty liver disease is associated with gut dysbiosis and shift in the metabolic function of the gut microbiota. Hepatology 2016; 63: 764-75.

13. Metchnikoff E. The prolongation of life: optimistic studies. William Heinemann, London 1907.

14. Yoo SR, Kim YJ, Park DY, et al. Probiotics L. plantarum and L. curvatus in combination alter hepatic lipid metabolism and suppress diet-induced obesity. Obesity (Silver Spring) 2013; 21: 2571-8.
15. Alisi A, Bedogni G, Baviera G, et al. Randomised clinical trial: the beneficial effects of VSL\#3 in obese children with non-alcoholic steatohepatitis. Aliment Pharmacol Ther 2014; 39: 1276-85.

16. Son G, Kremer M, Hines IN. Contribution of gut bacteria to liver pathobiology. Gastroenterol Res Pract 2010; 2010: pii: 453563.

17. Federico A, Dallio M, Godos J, Loguercio C, Salomone F. Targeting gut-liver axis for the treatment of nonalcoholic steatohepatitis: translational and clinical evidence. Transl Res J Lab Clin Med 2016; 167: 116-24.

18. Paolella G, Mandato C, Pierri L, Poeta M, Di Stasi M, Vajro P. Gut-liver axis and probiotics: their role in non-alcoholic fatty liver disease. World J Gastroenterol 2014; 20: 15518-31.

19. Vajro P, Paolella G, Fasano A. Microbiota and gut-liver axis: their influences on obesity and obesity-related liver disease. J Pediatr Gastroenterol Nutr 2013; 56: 461-8.

20. Hawrelak JA, Myers SP. The causes of intestinal dysbiosis: a review. Altern Med Rev J Clin Ther 2004; 9: 180-97.

21. Bertelsen RJ, Jensen ET, Ringel-Kulka T. Use of probiotics and prebiotics in infant feeding. Best Pract Res Clin Gastroenterol 2016; 30: 39-48.

22. Boltin D. Probiotics in Helicobacter pylori-induced peptic ulcer disease. Best Pract Res Clin Gastroenterol 2016; 30: 99-109.

23. Boursier J, Diehl AM. Nonalcoholic fatty liver disease and the gut microbiome. Clin Liver Dis 2016; 20: 263-75.

24. Derikx LAAP, Dieleman LA, Hoentjen F. Probiotics and prebiotics in ulcerative colitis. Best Pract Res Clin Gastroenterol 2016; 30: 55-71.

25. Hendaus MA, Jomha FA, Ehlayel M. Allergic diseases among children: nutritional prevention and intervention. Ther Clin Risk Manag 2016; 12: 361-72.

26. Lahner E, Bellisario C, Hassan C, Zullo A, Esposito G, Annibale B. Probiotics in the treatment of diverticular disease. A systematic review. J Gastrointest Liver Dis 2016; 25: 79-86.

27. Lichtenstein L, Avni-Biron I, Ben-Bassat O. Probiotics and prebiotics in Crohn's disease therapies. Best Pract Res Clin Gastroenterol 2016; 30: 81-8.

28. Lichtenstein L, Avni-Biron I, Ben-Bassat O. The current place of probiotics and prebiotics in the treatment of pouchitis. Best Pract Res Clin Gastroenterol 2016; 30: 73-80.

29. Ollech JE, Shen NT, Crawford CV, Ringel Y. Use of probiotics in prevention and treatment of patients with Clostridium difficile infection. Best Pract Res Clin Gastroenterol 2016; 30: 111-8.

30. Vernocchi P, Del Chierico F, Fiocchi AG, et al. Understanding probiotics' role in allergic children: the clue of gut microbiota profiling. Curr Opin Allergy Clin Immunol 2015; 15: 495-503.

31. Yoo JY, Kim SS. Probiotics and prebiotics: present status and future perspectives on metabolic disorders. Nutrients 2016; 8: 173.

32. Del Chierico F, Gnani D, Vernocchi P, et al. Meta-omic platforms to assist in the understanding of NAFLD gut microbiota alterations: tools and applications. Int J Mol Sci 2014; 15: 684-711.

33. Brunt EM, Kleiner DE, Wilson LA, Belt P, NeuschwanderTetri BA; NASH Clinical Research Network (CRN). Nonalcoholic fatty liver disease (NAFLD) activity score and the histopathologic diagnosis in NAFLD: distinct clinicopathologic meanings. Hepatology 2011; 53: 810-20.

34. Malaguarnera M, Vacante M, Antic T, et al. Bifidobacterium longum with fructo-oligosaccharides in patients 
with non alcoholic steatohepatitis. Dig Dis Sci 2012; 57: 545-53.

35. Ren T, Huang C, Cheng M. Dietary blueberry and bifidobacteria attenuate nonalcoholic fatty liver disease in rats by affecting SIRT1-mediated signaling pathway. Oxid Med Cell Longev 2014; 2014: 469059.

36. Seki E, Schnabl B. Role of innate immunity and the microbiota in liver fibrosis: crosstalk between the liver and gut. J Physiol 2012; 590: 447-58.

37. Xin J, Zeng D, Wang $\mathrm{H}$, et al. Preventing non-alcoholic fatty liver disease through Lactobacillus johnsonii BS15 by attenuating inflammation and mitochondrial injury and improving gut environment in obese mice. Appl Microbiol Biotechnol 2014; 98: 6817-29.

38. Sohn W, Jun DW, Lee KN, et al. Lactobacillus paracasei induces M2-dominant Kupffer cell polarization in a mouse model of nonalcoholic steatohepatitis. Dig Dis Sci 2015; 60: 3340-50.

39. An HM, Park SY, Lee DK, et al. Antiobesity and lipid-lowering effects of Bifidobacterium spp. in high fat diet-induced obese rats. Lipids Health Dis 2011; 10: 116.

40. Yin YN, Yu QF, Fu N, Liu XW, Lu FG. Effects of four Bifidobacteria on obesity in high-fat diet induced rats. World J Gastroenterol 2010; 16: 3394-401.

41. Borchers AT, Selmi C, Meyers FJ, Keen CL, Gershwin ME. Probiotics and immunity. J Gastroenterol 2009; 44: 26-46.

42. Glenny AM, O'Meara S, Melville A, Sheldon TA, Wilson C. The treatment and prevention of obesity: a systematic review of the literature. Int J Obes Relat Metab Disord 1997; 21: 715-37.

43. Casas IA, Dobrogosz WJ. Validation of the probiotic concept: Lactobacillus reuteri confers broad-spectrum protection against disease in humans and animals. Microb Ecol Health Dis 2000; 12: 247-85.

44. Wang J, Zhang H, Chen X, Chen Y, Menghebilige, Bao Q. Selection of potential probiotic lactobacilli for cholesterol-lowering properties and their effect on cholesterol metabolism in rats fed a high-lipid diet. J Dairy Sci 2012; 95: 1645-54.

45. Honda K, Moto M, Uchida N, He F, Hashizume N. Anti-diabetic effects of lactic acid bacteria in normal and type 2 diabetic mice. J Clin Biochem Nutr 2012; 51: 96-101. 